\title{
Enforcement Leverage with Fixed Inspection Capacity
}

\author{
Lirong Liu* \\ William Neilson ${ }^{\dagger}$
}

\begin{abstract}
We expand the optimal targeting enforcement literature to allow regulator inspection capacity constraints. A fixed number of firms are selected for inspection and those with the highest emissions are targeted with higher inspection probability. This structure induces dynamic rank-order tournaments among inspected firms, and pollution abatement incentives from the leverage effect are enhanced by a competition effect. Simulations suggest that targeted firms should be inspected with high probability and that about $2 / 3$ of inspections should be allocated to targeted firms. However, even suboptimal allocations of inspections and firms to the targeted and untargeted groups can outperform static enforcement schemes.
\end{abstract}

JEL Classification: D62; L51; Q58

Keywords: Monitoring and Enforcement; Environmental regulation; Targeting; Leveraging; Rank-order tournament.

\footnotetext{
* Department of Economics and International Business, Sam Houston State University, Huntsville, TX, 77341; email address: 1x1004@shsu.edu; 936-294-4231 (phone); 936-294-3488 (fax).

† Department of Economics, University of Tennessee, Knoxville, TN 37996-0550; e-mail address: wneilson@utk.edu; 865-974-1691 (phone); 865-974-4601 (fax).

We would like to thank Jon Harford, Mary Evans, Scott Gilpatric, Darren Grant, Donald Freeman, Per Fredriksson as well as seminar participants at the University of Tennessee and Sam Houston State University for their helpful comments.
} 


\section{Introduction}

It has long been known that regulators can receive more bang for their regulatory buck by leveraging their enforcement in the following manner, as described by Harrington (1988). Firms are placed into two groups based on their compliance status, with one group facing higher sanctions and higher inspection probabilities than the other group. Firms move from the lowenforcement (untargeted) group to the high-enforcement (targeted) group when they are found in violation of the regulatory standard, and move back to the untargeted group when they are found in compliance. This regulatory scheme has been alternately called leveraged enforcement, targeted enforcement, and state-dependent enforcement. Harrington (1988) shows that leveraged enforcement can be superior to static enforcement, in which all firms face the same inspection probabilities and sanction magnitudes. Others have extended Harrington's model to more general settings and more general regulatory structures, including Russell (1990), Harford (1991), Harford and Harrington (1991), Raymond (1999), and Stafford (2008). Friesen (2003) even shows that assigning firms to the targeted group randomly rather than based on a violation outperforms static enforcement.

These models establish that leveraged enforcement is a superior regulatory scheme, but they abstract away from one real-world aspect of regulatory agencies: constraints on the number of inspections the agency can perform. For example, the US Environmental Protection Agency (EPA) faces constrained fiscal budgets and workforce limitations, rendering impossible inspections of every firm every year. Specifically, between September 2006 and August 2007 in Region 4, only about $40 \%$ of the firms registered with hazardous waste management programs, about half of the firms registered with air programs, and about $75 \%$ of the firms registered with water programs were inspected at least once. ${ }^{1}$ In fact, the 2004 Strategic Plan of Region 4 states, "The vast number of regulated facilities in the region dictates that Region 4 prioritize where we devote our limited resources...the region has far more areas of critical concern than resources."2

\footnotetext{
${ }^{1}$ EPA Region 4 includes Alabama, Florida, Georgia, Kentucky, Mississippi, North Carolina, South Carolina, and Tennessee. Information is summarized according to Enforcement and Compliance History Online (ECHO) data; the data can be found at http://www.epa-echo.gov/echo/.

${ }^{2}$ U.S. EPA (2004,Chapter 2, goal 5, p. 1).
} 
Inspection capacity constraints create difficulties for traditional leveraged enforcement schemes. In these schemes, a firm is assigned to the targeted or untargeted group irrespective of the assignments of other firms. If too many firms are assigned to the targeted group the agency must exceed its inspection capacity to carry on with the targeted enforcement plan, while if too many are assigned to the untargeted group some of the agency's inspection capacity sits idle. When inspection capacity constraints are binding, though, the agency can only add a firm to the targeted group when it takes another firm out. This paper considers the optimal leveraged enforcement strategy for an agency facing binding inspection capacity constraints.

To do this, we construct a dynamic model that is similar in nature to those in the standard leveraged enforcement literature, with some firms placed in the targeted group and the rest in an untargeted group. Binding inspection capacity constraints impose two further restrictions. First, some of the inspections must be allocated to the targeted group, and the rest allocated to the untargeted group, with at least one inspection allocated to each group. Second, when inspection capacity is constant through time, the number of firms moving out of each group in the current period must equal the number of firms moved into that group in the next period. Making a firm's transition probability from one group to the other dependent upon its compliance status alone no longer satisfies that requirement. Thus we assume that the inspected firms compete with each other for the chance of being placed in the untargeted group. The structure of this transition process induces rank-order tournaments among inspected firms, and the tournament actually enhances the impact of leveraged enforcement. ${ }^{3}$

To understand how leveraging and the tournament structure work together, consider a pollution emissions example in which there are 10 total firms and the regulator has the capacity to inspect 4 of them each period. For the sake of discussion, suppose that the regulator places 2 firms in the targeted group and 8 in the untargeted group, and allocates 2 inspections to the targeted group and 2 to the untargeted group. This means that firms in the targeted group are certain to be inspected, while firms in the untargeted group face only a $1 / 4$ chance of inspection.

\footnotetext{
${ }^{3}$ Tournament models have been widely used in the study of labor economics and other related fields since the pioneering work by Lazear and Rosen (1981), but applications of tournament models in environmental economics are quite limited. See Govindasamy, Herriges, and Shogren (1994), and Franckx, D'Amato and Brose (2004) for examples.
} 
Also, among the four inspected firms the two with the highest emissions are assigned to the targeted group next period, and the two with the lowest emissions are assigned to the untargeted group. Now suppose that the regulator increases both the size and the inspection capacity of the targeted group to 3 , reducing the untargeted group to 7 firms with only 1 inspection. Targeted firms are impacted in two ways. First, the cost differential between the two groups has increased because being in the untargeted group has become much better. This makes moving out of the targeted group more attractive, essentially increasing the prize for winning the tournament, and firms abate more because of this increased cost differential. This is the leverage effect, and it is present in traditional leveraged enforcement models. The second impact comes because of the change in the nature of the tournament. Before 2 targeted firms competed with 2 untargeted firms for 2 spots in the untargeted group. Now 3 targeted firms compete with 1 untargeted firm for only 1 spot in the untargeted group. This enhanced competition leads to further abatement and we call it the competition effect. The competition effect is new to this paper.

The complex nature of the problem allows for only limited analytical results, and most of our analysis is performed using numerical simulations. We find four main results. Leveraged enforcement, even with suboptimal allocations of inspections and firms to groups, outperforms static enforcement. Leveraged enforcement works best when firms in the targeted group are inspected with certainty, and about $2 / 3$ of the inspections should be allocated to the targeted group. Even though a small minority of firms are in the targeted group, most of the abatement activity comes from this group. Finally, even though the capacity constraint imposes the tournament structure leading to the competition effect, relaxing the constraint still leads to more abatement effort and lower emissions.

The paper is organized as follows. The model is developed in Sections II and III, with the decisions of firms considered in Section II and the regulator's problem considered in Section III. The problem is framed within an environmental economics setting, with firms abating to meet an emissions standard set by the regulator, but can be generalized to other regulatory settings. Section IV contains the numerical simulations along with most of our results. Section V offers a summary and conclusion. 


\section{Firm behavior under dynamic enforcement}

Consider a total of $n$ homogenous firms with identical abatement functions and abatement cost functions. Every firm faces a standard $s$ above which excess emissions are penalized with a fixed fine $\gamma$. Let a firm's measured emissions be denoted as $z=T-e+\varepsilon$, where $e$ is the firm's abatement effort level, $T$ is the firm's fixed total emissions, and $\varepsilon$ is a random error term that is independently and identically distributed across all firms with mean zero, density function $f(\varepsilon)$, and distribution function $F(\varepsilon)$. The probability that a firm with abatement effort $e$ is found out of compliance can be written as

$$
Q(e)=\operatorname{Pr}\{z>s\}=\operatorname{Pr}\{T-e+\varepsilon>s\}=1-F(s-T+e) .
$$

When a firm is inspected, it also incurs a fixed cost, denoted $\alpha$. The fixed cost represents the pecuniary and nonpecuniary costs borne by the firm other than the abatement costs, such as those associated with paperwork preparations for inspection. The firm's total cost in a single period can be written as

$$
\mu=c(e)+\rho[\gamma Q(e)+\alpha],
$$

where $c(e)$ is the abatement cost function and $\rho$ is the probability that the firm is inspected.

In a leveraged or targeting enforcement regime, the $n$ firms are classified into two groups, 1 and 2, where group 2 is the targeted group with tougher enforcement. To keep the model simple and to isolate the impact of group assignment, we assume that the only difference in the treatment of the two groups is the probability of inspection, which is higher in group 2 than in group 1 . The penalty for violation, the fixed inspection cost and the standard are the same for all firms regardless of their group status.

Let $n_{1}$ and $n_{2}$ denote group sizes, where $n_{1}+n_{2}=n$. In each period, a total of $m(3 \leq m \leq$ $n-1)$ firms are inspected, with $m_{1}$ of them randomly selected from group 1 and $m_{2}$ from group 2. ${ }^{4}$ The number of inspections $m$ is exogenously fixed by the inspection capacity. Note that $\rho_{1}=$ $m_{1} / n_{1}$ and $\rho_{2}=m_{2} / n_{2}$ are the inspection probabilities in each group, and we restrict $\rho_{2}>\rho_{1}$ so that group 2 is the targeted group. Of the $m_{1}+m_{2}$ inspected firms, the $m_{1}$ firms with the lowest

\footnotetext{
${ }^{4}$ Three is the minimum number of firms for which leverage is possible.
} 
measured emissions in period $t$ are placed in group 1 for period $t+1$, and the $m_{2}$ firms with the highest emissions are placed in group 2. If a firm is not inspected in a specific period, it stays in the same group.

The structure of this transition process induces rank-order tournaments among the inspected firms. In the tournament, the probability that a firm wins is a function of its own effort level as well as the effort levels of other inspected firms. Even if a firm is found to be in compliance with the standard, it may nevertheless end up in group 2 in the next period if its emissions are among the $m_{2}$ highest. Similarly, a noncompliant firm may be placed in group 1 if the emission levels of other firms turn out to be higher. In the symmetric equilibrium, firms in the same group exert the same optimal effort, and firm-specific subscripts can be replaced by group-specific subscripts. The probability that an inspected firm from group $i$ ends up in group 2 in the next period can be denoted as $p_{i}\left(e_{i}, e_{-i}, e_{j}\right)$, where $e_{i}$ and $e_{-i}$ are the effort levels of this specific firm and other firms in the same group, respectively, and $e_{j}$ is the effort level of firms in the other group. As higher effort increases the probability that a firm wins the tournament, it follows that $\partial p_{i}\left(e_{i}, e_{-i}, e_{j}\right) / \partial e_{i}<0$.

For any firm in this regulation scheme, its decision involves choosing the level of abatement effort to minimize the expected present value (EPV) of the total cost in all periods. The firm's decision follows a Markov chain process with the transition matrix describing the probabilities of firms moving from one group to the other shown in Table 1 (the arguments in $p_{i}$ 's are omitted).

\section{Table 1. Markov transition matrix}

\begin{tabular}{|c|c|c|}
\hline & \multicolumn{2}{|c|}{ To Group } \\
\hline From Group & 1 & 2 \\
\hline 1 & $1-\rho_{1} p_{1}$ & $\rho_{1} p_{1}$ \\
\hline 2 & $\rho_{2}\left(1-p_{2}\right)$ & $1-\rho_{2}\left(1-p_{2}\right)$ \\
\hline
\end{tabular}

Let $V_{i t}$ denote the EPV of the total cost for a firm starting from group $i$ in period $t$. It follows that 


$$
\begin{gathered}
V_{1 t}=\mu_{1 t}+\delta\left(1-\rho_{1 t} p_{1 t}\right) V_{1, t+1}+\delta \rho_{1 t} p_{1 t} V_{2, t+1}, \\
V_{2 t}=\mu_{2 t}+\delta \rho_{2 t}\left(1-p_{2 t}\right) V_{1, t+1}+\delta\left[1-\rho_{2 t}\left(1-p_{2 t}\right)\right] V_{2, t+1},
\end{gathered}
$$

where $\delta$ is the discount factor. These equations state that the EPV of the total cost for a firm is the sum of its current period cost and the discounted EPV of the total cost starting from the next period. The firm chooses the optimal effort levels to minimize $V_{i t}$. According to the ergodic theorem of Markov chains, the optimal strategy for a firm is stationary (Harrington, 1988; Kohlas, 1982). Therefore, the time subscript $t$ is dropped from what follows.

Assuming an interior solution, the first order condition for this optimization problem is

$$
\frac{\partial \mu_{i}}{\partial e_{i}}=-\delta\left(V_{2}-V_{1}\right) \rho_{i} \frac{\partial p_{i}}{\partial e_{i}}
$$

Equation (5) characterizes the optimal effort level of the firms in each group, $e_{i}^{*}\left(m_{i}, n_{i}, \gamma, \alpha, s\right)$. The left-hand side of the equation is the marginal change in the current period cost, while the right-hand side represents the marginal decrease in the EPV of the total cost as a higher $e_{i}$ reduces the probability of being in group 2 in the next period. The first-order condition shows the willingness of a firm to incur higher costs in the current period in exchange for the expected savings resulting from a decreased probability of facing tougher enforcement in the future. The optimal effort level for any firm should be the one that equates the marginal change in oneperiod cost to the discounted savings on the expected future cost.

Notice that $V_{2}-V_{1}$ is the cost differential between firms starting from group 1 versus group 2, and it can be solved from equations (3) and (4) to be

$$
V_{2}-V_{1}=\frac{\mu_{2}-\mu_{1}}{1-\delta\left[1-\rho_{2}\left(1-p_{2}\right)-\rho_{1} p_{1}\right]}>0
$$

Thus in equation (5), the only negative term on the right hand side is $\partial p_{i} / \partial e_{i}$. It follows that $\partial \mu_{i} / \partial e_{i}{ }^{*}>0$, where $e_{i}{ }^{*}$ is a firm's optimal effort level when it is placed in group $i$. For a convex cost function $\mu_{i}$, this implies that $e_{i}^{*}$ is higher than the optimal effort level under static enforcement, denoted $\widetilde{e}_{i}$, which satisfies $\partial \mu_{i} / \partial \widetilde{e}_{i}=0$. This condition reveals one of the 
advantages of leveraged enforcement: firms in both groups have an extra incentive to increase abatement effort levels. Differentiating the EPV of the total cost in the two groups, creates leverage effects on a firm's emissions and abatement levels. Firms in both groups, anticipating the threat of being in group 2 and facing the higher inspection probability in the next period, exert more effort.

It is easy to show that $e_{2}{ }^{*}>e_{1}{ }^{*}$ must hold. ${ }^{5}$ In fact, this is an expected result of targeting enforcement. When a firm is in group 2, it is at a disadvantage as the EPV of its total cost is higher than the EPV of the total cost for firms in group 1. Therefore, this firm should exert more effort to secure a higher probability of winning in the tournament. On the other hand, firms in group 1 face a lower inspection frequency and exert less effort.

\section{The regulator's monitoring and enforcement strategies}

Now consider a regulator who is responsible for monitoring $n$ firms and enforcing the standard. The potential policy instruments at his/her disposal include the inspection frequency, which is determined by the allocation of inspections, the standard, and the penalty for violation. ${ }^{6}$ However, to emphasize the structure of enforcement with fixed inspection capacity, we consider the case in which the only choice variable for the regulator is the inspection frequency.

Recall that the inspection probabilities are defined as $\rho_{1}=m_{1} / n_{1}$ and $\rho_{2}=m_{2} / n_{2}$. The regulator's objective is to optimally allocate the inspections to each group and determine the sizes of the two groups to minimize the total emissions of all firms, with the assumption that this total emission level is not below the socially optimal level. ${ }^{7}$ Also, by construction, minimizing total emissions is equivalent to maximizing total effort. Formally, the regulator's objective is to

\footnotetext{
${ }^{5}$ The only way for $e_{2}{ }^{*}=e_{1}{ }^{*}$ to hold would be for $\rho_{2}=\rho_{1}$, which would mean that both groups were inspected with equal probability. When group 2 is targeted, though, it must be the case that $\rho_{2}>\rho_{1}$, implying that $e_{2}{ }^{*}>e_{1}{ }^{*}$.

${ }^{6}$ Although the regulator may also have some influence on the fixed cost borne by the inspected firms and the variance of the error term, it is more likely that these parameters are beyond the control of the regulator.

${ }^{7}$ Theoretically the social optimal emission level is determined by the social benefits and social costs of emissions, which, in turn, determine the standard. Viscusi and Zeckhauser (1979) and Jones (1989) address the issue of standard setting under incomplete enforcement. Since the discussion of environmental standards is beyond the scope of this paper, we simply assume that the minimum total emissions from the optimal inspection strategy do not fall below the social optimal emission level so that the optimal leverage is desirable.
} 


$$
\max _{m_{i}, n_{i}} n_{1} e_{1}^{*}+n_{2} e_{2}^{*}
$$

To get a feel for the regulator's problem, consider a regulator allocating 10 inspections among 100 firms. To describe the trends of firm effort under different policy choices, we start with an extreme case where there is only one member in group 2; that is, $m_{2}=n_{2}=1, m_{1}=9$, and $n_{1}=99$. In the resulting tournament, nine group-1 firms and one group-2 firm compete in period $t$ for the chance of being placed in group 1 in period $t+1$, with 9 "winners" and one "loser." The group-2 firm can be regarded as a strong competitor because its abatement effort is high, while the group-1 firms are relatively weak competitors with lower abatement effort. Now suppose the regulator allocates another inspection and another firm to group 2, so that $m_{2}$ and $n_{2}$ increase to 2, $m_{1}$ falls to 8 , and $n_{1}$ falls to 98 . The firms in group 2 increase their effort due to two forces. The first is the leverage effect. Moving an inspection to group 2 from group 1 reduces the probability of inspection in group 1 from 0.09 to 0.08 , making group 1 membership more valuable. Consequently, it is optimal for group- 2 firms to abate more in order to improve their chances of winning in the tournament. The second force is a result of the competition effect. When a single group-2 firm competes with nine group-1 firms, the group-2 firm has a high chance of winning the tournament because group-2 firms abate more than group-1 firms do. When a second firm is added to group 2, though, the group-2 firms must compete with each other as well as the eight group-1 firms, and this head-to-head competition among the group-2 firms, who are the strong competitors, leads to higher abatement levels.

The two forces also impose similar effects on the effort of firms in group 1, but a third, countervailing force means that the overall change in the abatement effort of group-1 firms may not necessarily increase. The reduced inspection probability in group 1 leads to a lower expected penalty for violation, which dissipates the incentive for group-1 firms to reduce emissions. We refer to this as an inspection effect, and the overall change in the effort of group-1 firms is generally ambiguous.

Another extreme case is to allocate all but one inspection to group 2, setting $m_{2}=n_{2}=9$, $m_{1}=1$, and $n_{1}=91$. Each period's tournament pits nine group-2 firms against one group-1 firm, and only the firm with the lowest emission level wins the tournament. If the regulator reduces $m_{2}$ and $n_{2}$ to 8 , two changes affect the effort levels: (1) the inspection probability in group 1 
increases from 0.01 to 0.02 and the cost differential decreases with it; (2) firms in the tournament compete with one more group-1 firm (one more weak competitor) and one fewer group-2 firm (one less strong competitor), making the competition less intensive. For group-1 firms the smaller cost differential and diminished competition suggest that reducing abatement effort is optimal, but the higher inspection probability induces group-1 firms to increase effort. Overall, the change in the effort of group-1 firms is ambiguous. On the other hand, group-2 firms lower abatement effort because of the smaller cost differential and the reduced competition. As a result of the interaction among firms, however, group-2 firms may still exert more effort in response if group-1 firms increase their effort.

The traditional optimization tools-first order conditions with respect to the choice variables - do not apply here because the choice variables take integer values only. Furthermore, as the examples above show, analytically comparing the total effort from all possible allocations to determine the optimal enforcement strategy is not feasible due to the complexity of the firm's problem. For these reasons we use simulations to explore the characteristics of the optimal allocation of inspections and group sizes.

\section{Simulations}

To characterize the optimal enforcement strategy, we use numerical techniques to identify the group assignments and allocations of inspections that result in the maximum total effort of all firms. Although the problem is complex because of the number of firms competing in an asymmetric tournament, the primitives of the problem are few in number and relatively simple. In particular, to run the simulations the only functions one need specify are the abatement cost function $c(e)$ and the distribution of the error term $\varepsilon$. Remaining parameters

include the emissions standard $s$, the maximal emissions level $T$ from which firms abate, the lump-sum penalty for violations $\gamma$, the fixed inspection $\operatorname{cost} \alpha$, and the discount factor $\delta$. Once these functions and parameters are specified, the general approach consists of computing equilibrium behavior for different total numbers of firms, different total numbers and allocations of inspections, and different group sizes. 
For the simulations the abatement cost function is specified as $c(e)=w e^{2}$, where $w$ is a positive parameter. As for the distribution assumptions of the error term, we use a normal distribution centered around zero. ${ }^{8}$ A desirable feature of a mean-zero normal distribution is that the peak of its density function occurs at the point where the measured emissions through inspection are equal to the firm's intended emissions. The parameters for the baseline simulation are listed in Table $2 .^{9}$

Table 2. Parameters for baseline simulation

\begin{tabular}{|l|c|}
\hline Parameter & Value \\
\hline Total emissions, $T$ & 2.5 \\
Standard, $s$ & 2.0 \\
Abatement cost function parameter, $w$ & 18.0 \\
Penalty for violation, $\gamma$ & 3.0 \\
Fixed inspection cost, $\alpha$ & 0.5 \\
Discount rate, $\delta$ & 0.9 \\
Standard deviation of the error term, $\sigma$ & 0.45 \\
\hline
\end{tabular}

We begin with a setting in which there are 10 firms and the regulator has the capacity to inspect 4 of them. Since $m_{1}$ and $m_{2}$ must both be strictly positive and sum to 4 , this leaves three possible combinations of $m_{1}$ and $m_{2}$. For each of these combinations, the regulator must decide how many of the 10 firms to place in group 2, with the remainder going in group 1, under the constraints that $n_{2} \geq m_{2}$ and $n_{1} \geq m_{1}$. Table 3 shows the total combined effort of all 10 firms under the different inspection and group assignment scenarios. ${ }^{10}$ Begin with the second column, in which the regulator assigns one inspection to group 1 and three inspections to group 2 . In these cases group 2 must contain at least three members, and group 1 can get as large as 7 members. Enlarging group 1 leads to higher total effort, and the maximum total effort arises when the targeted group is as small as possible, so that the inspection probability in group 2 is

\footnotetext{
${ }^{8} \mathrm{~A}$ uniform distribution generates qualitatively similar results, which can be found in Appendix B.

${ }^{9}$ Empirical studies suggest that abatement costs are high compared to penalties and other sanctions. For example, the Pollution Abatement Costs and Expenditures Survey (U.S. Bureau of the Census, 1999) reveals that the total abatement cost across all industries amounts to $\$ 5.8$ billion, while total payment to the government, including permits/fees and charges, fines/penalties, and other, is only $\$ 1.0$ billion. Because of this the abatement cost parameter $w$ is set higher than the other parameters in the baseline treatment.

${ }^{10}$ The simulation fails to converge when group 2 was too large and group 1 was too small, in which case the cell is left blank.
} 
one. Furthermore, the largest jump in effort comes when the inspection probability in the targeted group increases to 1 .

The third and fourth columns show different allocations of the inspections across groups. In both of those columns, total effort is maximized when the inspection probability for the targeted group is 1 . Comparing across the columns, we see that total effort is maximized when $m_{1}=1, m_{2}=n_{2}=3$, and $n_{1}=7$; that is, when all but one inspection is allocated to the targeted group and the inspection probability in the targeted group is one.

Table 3. Baseline results for all possible allocations: 4 inspections for 10 firms

\begin{tabular}{|c|c|c|c|c|c|c|c|c|c|}
\hline & \multicolumn{3}{|c|}{$m_{1}=1, m_{2}=3$} & \multicolumn{3}{c|}{$m_{1}=2, m_{2}=2$} & \multicolumn{3}{c|}{$m_{1}=3, m_{2}=1$} \\
\hline$n_{1}$ & Total effort & $\rho_{1}$ & $\rho_{2}$ & Total effort & $\rho_{1}$ & $\rho_{2}$ & Total effort & $\rho_{1}$ & $\rho_{2}$ \\
\hline 3 & 0.1880 & $1 / 3$ & $3 / 7$ & -- & $2 / 3$ & $2 / 7$ & -- & 1 & $1 / 7$ \\
\hline 4 & 0.2330 & $1 / 4$ & $1 / 2$ & -- & $1 / 2$ & $1 / 3$ & -- & $3 / 4$ & $1 / 6$ \\
\hline 5 & 0.2928 & $1 / 5$ & $3 / 5$ & -- & $2 / 5$ & $2 / 5$ & -- & $3 / 5$ & $1 / 5$ \\
\hline 6 & 0.4028 & $1 / 6$ & $3 / 4$ & 0.2426 & $1 / 3$ & $1 / 2$ & -- & $1 / 2$ & $1 / 4$ \\
\hline 7 & 0.7850 & $1 / 7$ & 1 & 0.3600 & $2 / 7$ & $2 / 3$ & -- & $3 / 7$ & $1 / 3$ \\
\hline 8 & -- & -- & -- & 0.6048 & $1 / 4$ & 1 & 0.1930 & $3 / 8$ & $1 / 2$ \\
\hline 9 & -- & -- & -- & -- & -- & -- & 0.3177 & $1 / 3$ & 1 \\
\hline
\end{tabular}

Table 4 parses the information in Table 3 further, showing how effort levels of firms in the different groups. Based on the trends in Table 3, we reduce the number of possible assignments by restricting that $m_{2}=n_{2}$, so that the probability of inspection in the targeted group is 1 . With this restriction, $n_{1}=n-m_{2}$ and $m_{1}=m-m_{2}$, and we reduce the problem with two choice variables to a problem with a single choice variable, $m_{2}$, and we retain this restriction through the remainder of the simulations. 
Table 4. Firm-level baseline results: 4 inspections for 10 firms

\begin{tabular}{|c|c|c|c|c|c|c|}
\hline$m_{2}$ & $e_{1}{ }^{*}$ & $e_{2}{ }^{*}$ & $n_{1} e_{1}{ }^{*}$ & $n_{2} e_{2}{ }^{*}$ & $n_{1} e_{1}{ }^{*}+n_{2} e_{2}{ }^{*}$ & $\rho_{1}$ \\
\hline 3 & 0.0129 & 0.2317 & 0.0900 & 0.6950 & 0.7850 & $1 / 7$ \\
\hline 2 & 0.0363 & 0.1571 & 0.2906 & 0.3142 & 0.6048 & $1 / 4$ \\
\hline 1 & 0.0267 & 0.0769 & 0.2407 & 0.0769 & 0.3177 & $1 / 3$ \\
\hline 0 & 0.0166 & -- & 0.1660 & -- & 0.1660 & $4 / 10$ \\
\hline
\end{tabular}

Note: inconsistencies of calculation are due to rounding errors.

Several patterns can be observed in Table 4. First, the random inspection strategy without leverage (corresponding to $m_{2}=0$ ) induces the least total effort, and therefore even suboptimal targeting is superior to static enforcement. Second, if an inspection is moved from group 1 to group 2, the effort of each group 2 firm increases, consistent with the leverage and competition effects, while the effort of group 1 firms may increase or decrease, consistent with the confounding presence of the inspection probability effect. More specifically, when $m_{2}$ increases, it creates more competition among firms in both groups, because a weak group-1 firm is replaced by a strong group- 2 firm in the tournament. In this example, the effort of a group- 2 firm increases steadily when $m_{2}$ increases from 1 to 3 . However, group-1 firms may exert less effort because the increase in $m_{2}$ lowers the inspection probability in group 1 (shown in the last column in Table 4). For example, the competition and leverage effects dominate for the group-1 firms when $m_{2}$ increases from 1 to 2 , but the inspection probability effect dominates when $m_{2}$ increases from 2 to 3 . Setting $m_{2}=3$ yields the highest total effort, and it occurs because the increased effort by group- 2 firms outweighs the decreased effort by group- 1 firms.

The primary result from the baseline example is that the regulator minimizes total emissions when it leverages its limited inspections by allocating most of them to the targeted group, and inspecting the targeted group with probability 1 . This allocation remains optimal even for different values of the penalty parameter $\gamma$, the fixed inspection $\operatorname{cost} \alpha$, and the standard deviation $\sigma$, as shown in Figure 1. Each panel in the figure shows the impact of changing a different parameter, but in every case assigning more inspections to group 2 results in increased abatement effort, and $m_{2}=3$ is the optimal allocation. Static enforcement $\left(m_{2}=0\right)$ always performs the worst. 
Figure 1. Robustness of the baseline simulation: 4 inspections for 10 firms
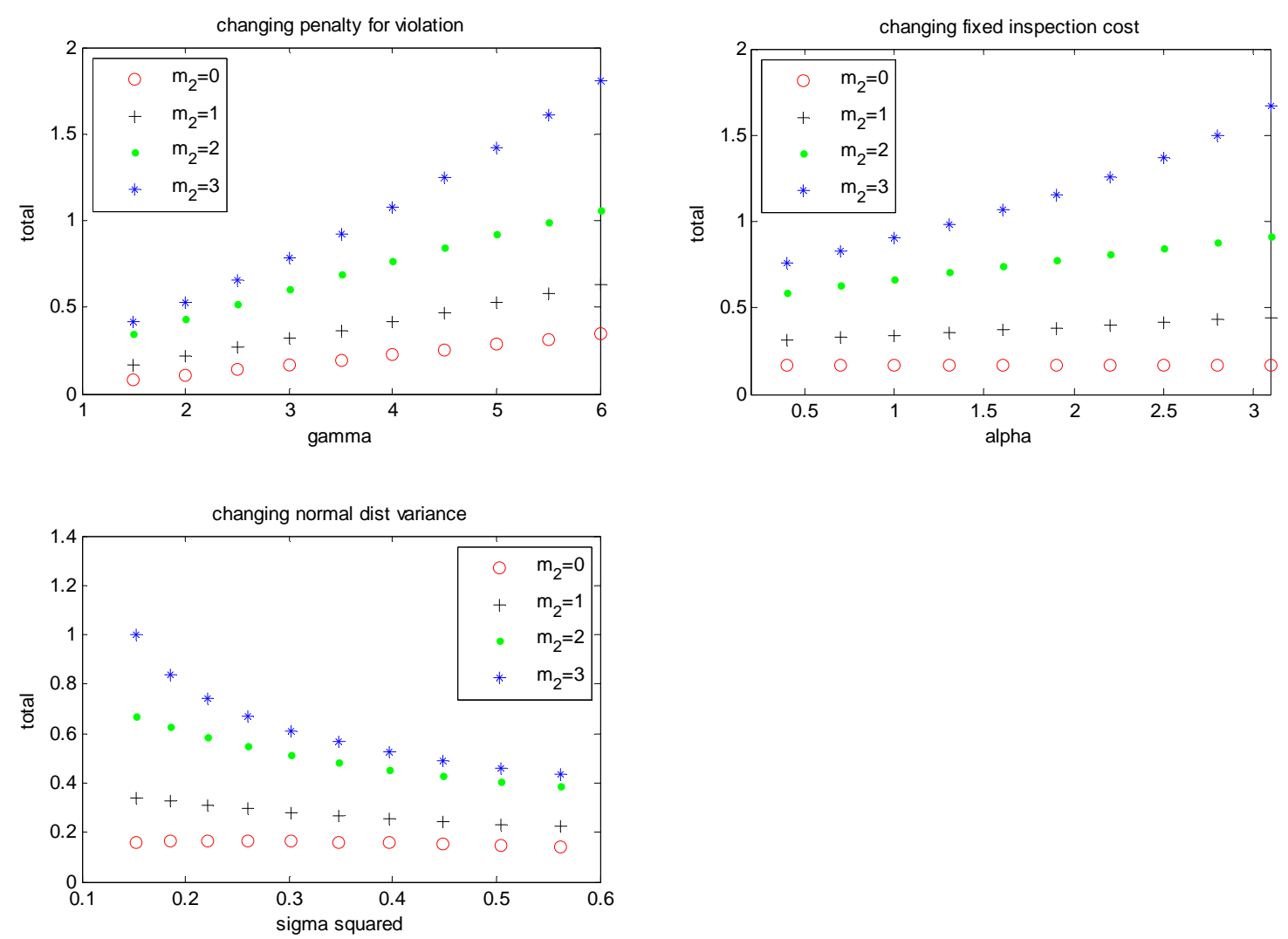

In all three cases abatement effort levels move in the intuitively plausible direction, at least at the aggregate level. Increasing the penalty for a violation gives firms in both groups an incentive to abate more, and total effort increases when $\gamma$ increases. Increasing the fixed inspection cost makes being in group 2 more costly, and so firms abate more to increase their chances of moving to group 1 , and effort increases as $\alpha$ increases. Increasing the standard deviation of the error term means that outcomes are less correlated with inputs, diluting the incentive to exert effort toward abatement. As the figure shows, increases in $\sigma$ lead to reductions in abatement effort. 


\section{Figure 2. Firm-level effects of changing the penalty}
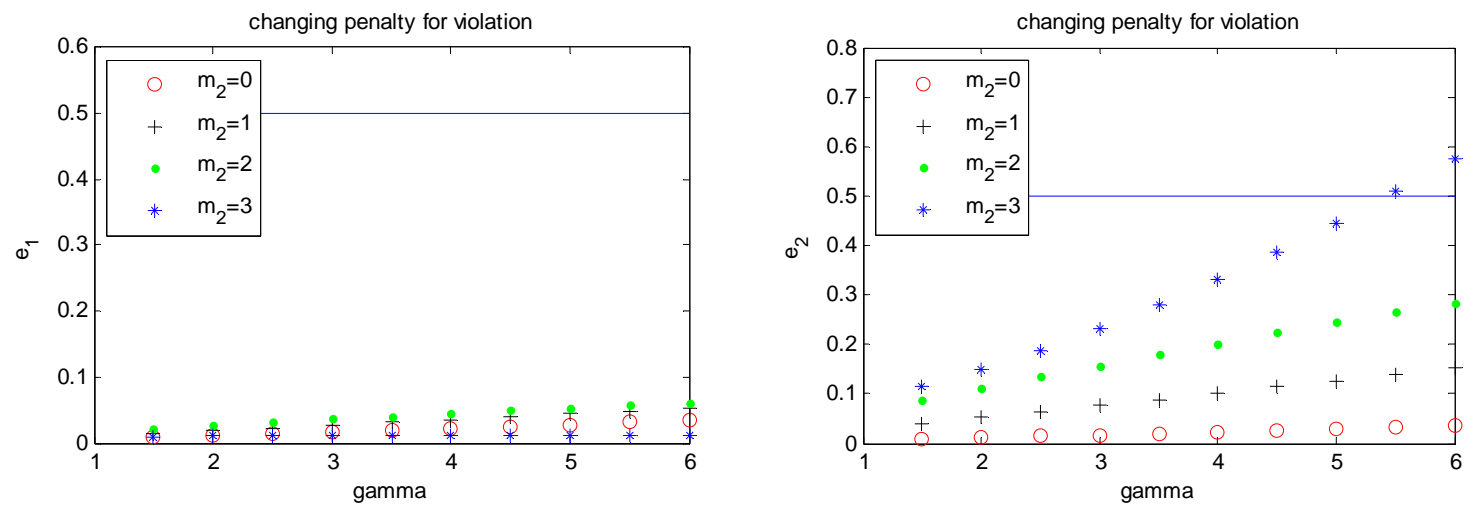

Figure 2 breaks this analysis down into its component parts for one of the three parameters, the size of the penalty for violations. The two panels of Figure 2 show the responses of a group-1 firm and a group- 2 firm to changes in $\gamma$. A horizontal line representing the difference between $T$ and $s$ is added. In expectation, a firm is in compliance if its effort is sufficient to eliminate the excess emissions above the standard (in the absence of random errors), which is $T-s$. Thus, effort levels above the horizontal line suggest that firms are overcomplying in expectation, and effort levels below this line imply undercompliance in expectation.

Several results follow from Figure 2, and they extend to the analysis of the other parameters, as well. First, aggregate effort levels are driven by the group-2 firms and not the group-1 firms, since group-2 effort levels are much larger than group-1 levels. Second, group-2 firms come closer to the emissions standard than group-1 firms, and group 2 firms even exceed the emissions standard for sufficiently high penalties. Third, while $m_{2}=3$ generates the most effort in aggregate and from the group-2 firms, a different allocation, $m_{2}=2$, returns more effort from group-1 firms. This occurs because the inspection effect matters for group-1 firms but not for group-2 firms, which are inspected with probability one.

So far we have the robust result that allocating more inspections to the targeted group is optimal. For an inspection capacity with $m=4$, the optimal allocation is $m_{2}=3$. Unfortunately, the three inspections allocated to group 2 can be interpreted in several different ways: as $1+m / 2$ (just over half of all inspections), $3 m / 4$ (three-fourths of all inspections), or $m-1$ (all but one 
inspection). The simple example of four inspections does not provide sufficient information to draw a conclusion whether the optimal number of inspections in group 2 should be around $m / 2$, $3 m / 4$, or $m-1$ for higher values of $m$. In the next set of simulations, we address this issue and check the consistency of other relevant results from the baseline analysis as well.

To do this we consider the case with 10 inspections for 100 firms. The standard deviation of the error term is set at 0.8 to ensure the existence of solutions. ${ }^{11}$ The optimal effort of individual firms and the total effort of all firms are shown in Table 5. For firms in group 1 effort peaks when $m_{2}=5$, and for firms in group 2 effort peaks when $m_{2}=7$. As before, aggregate behavior is dominated by the firms in group 2, and total effort peaks when $m_{2}=7$. It is clear from this example that neither $m_{2}=1+m / 2$ nor $m_{2}=m-1$ are optimal. Instead, the optimal number of inspections allocated to group 2 is between $m / 2$ and $3 m / 4$. Also, as before, static enforcement with $m_{2}=0$ yields the lowest levels of total effort.

Table 5: 10 inspections for 100 firms

\begin{tabular}{|c|c|c|c|c|c|}
\hline$m_{2}$ & $e_{1}{ }^{*}$ & $e_{2}{ }^{*}$ & $n_{1} e_{1}{ }^{*}$ & $n_{2} e^{*}$ & $n_{1} e_{1}{ }^{*}+n_{2} e_{2}{ }^{*}$ \\
\hline 9 & 0.0005 & 0.0462 & 0.0413 & 0.4158 & 0.4572 \\
\hline 8 & 0.0015 & 0.1160 & 0.1390 & 0.9280 & 1.0668 \\
\hline 7 & 0.0030 & $\mathbf{0 . 2 7 7 4}$ & 0.2790 & $\mathbf{1 . 9 4 1 8}$ & $\mathbf{2 . 2 2 0 7}$ \\
\hline 6 & 0.0063 & 0.2465 & 0.5906 & 1.4790 & 2.0696 \\
\hline 5 & $\mathbf{0 . 0 0 8 4}$ & 0.1623 & $\mathbf{0 . 7 9 3 3}$ & 0.8115 & 1.6046 \\
\hline 4 & 0.0072 & 0.1021 & 0.6951 & 0.4084 & 1.1034 \\
\hline 3 & 0.0050 & 0.0627 & 0.4851 & 0.1881 & 0.6732 \\
\hline 2 & 0.0035 & 0.0423 & 0.3477 & 0.0846 & 0.4324 \\
\hline 1 & 0.0031 & 0.0359 & 0.3106 & 0.0359 & 0.3465 \\
\hline 0 & 0.0034 & -- & 0.3428 & -- & 0.3428 \\
\hline
\end{tabular}

Notes: 1. inconsistencies of calculation are due to rounding errors; 2. bold numbers indicate the maximum within each column.

Figure 3 shows how the optimal allocation changes when the parameters $\gamma, \alpha$, and $\sigma$ change. In all cases the optimal value of $m_{2}$ is either 7 or 6 , with more inspections optimally allocated to group 2 when penalties are larger, fixed inspection costs are higher, or measurements

\footnotetext{
${ }^{11}$ The existence of solutions requires that the variance is sufficiently large. See Lazear and Rosen (1981), footnote 2, p. 845, and also Nalebuff and Stiglitz (1983).
} 
are more precise. Furthermore, the parameters lead to changes in the same directions as in the baseline case shown in Figure 1.

Figure 3. Robustness check: 10 inspections for 100 firms
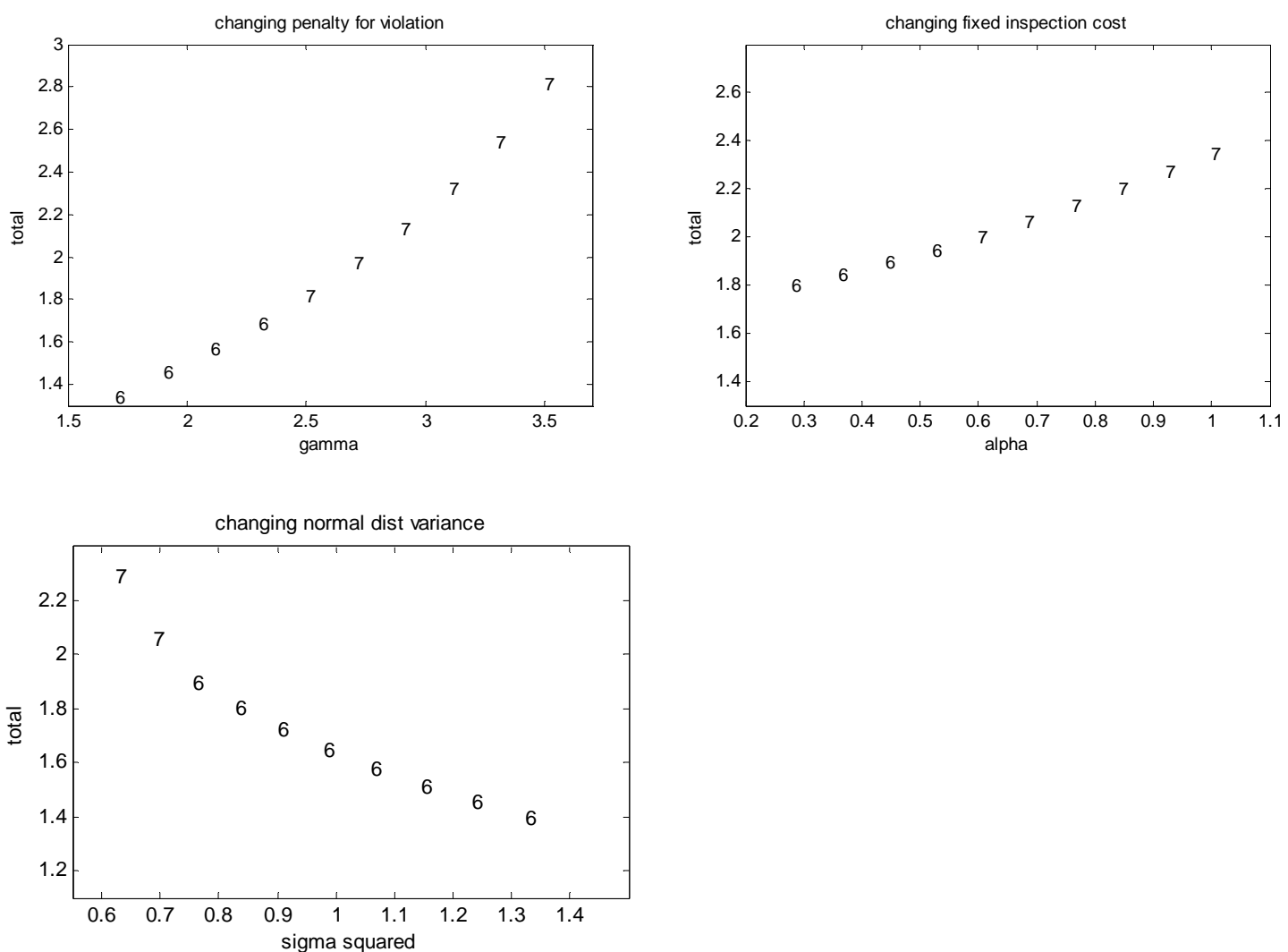

The final set of simulations holds the total number of firms constant but changes the number of inspections available, and Table 6 shows the total abatement effort levels when there are 25 firms, with boldface signifying the highest abatement levels in each row. ${ }^{12}$ As the table shows, sometimes additional inspection capacity is best utilized by expanding the targeted group 2 , and sometimes it is best utilized by increasing the inspections of group 1. The fraction of inspections allocated to group 2 lies consistently between 60 and 75 percent of the inspection

\footnotetext{
${ }^{12}$ Appendix A provides a similar table for 100 firms, with qualitatively similar results.
} 
capacity. Importantly, though, throughout the range of capacities shown, expanding inspection capacity for the regulator leads to increased abatement effort on the part of the firms.

Table 6. Total abatement effort levels, 25 firms

\begin{tabular}{|c|c|c|c|c|c|c|c|c|c|}
\hline & \multicolumn{7}{|c|}{ Number of inspections in group 2 } \\
\hline $\begin{array}{c}\text { Total number } \\
\text { of inspections }\end{array}$ & 1 & 2 & 3 & 4 & 5 & 6 & 7 & 8 & 9 \\
\hline 4 & 0.2345 & 0.4383 & $\mathbf{0 . 5 3 5 6}$ & & & & & & \\
\hline 5 & 0.2454 & 0.4542 & $\mathbf{0 . 6 7 2 7}$ & 0.5970 & & & & & \\
\hline 6 & 0.2606 & 0.4450 & 0.7191 & $\mathbf{0 . 8 7 3 5}$ & 0.5696 & & & & \\
\hline 7 & 0.2653 & 0.4273 & 0.7288 & 0.8588 & $\mathbf{0 . 9 7 7 2}$ & 0.5117 & & & \\
\hline 8 & 0.2972 & 0.4123 & 0.7338 & 0.4206 & $\mathbf{1 . 1 8 1 3}$ & 0.9468 & 0.4613 & & \\
\hline 9 & 0.3237 & 0.4055 & 0.6269 & 0.9602 & 1.2481 & $\mathbf{1 . 2 7 5 3}$ & 1.2408 & 0.4314 & \\
\hline 10 & 0.3480 & 0.4087 & 0.584 & 0.8986 & 1.2428 & $\mathbf{1 . 4 2 9 7}$ & 1.2408 & 0.7361 & 0.4218 \\
\hline
\end{tabular}

The above tables and figures show the effects of parameter changes on equilibrium behavior, but they do not show out-of-equilibrium behavior, in particular how firms respond to each others' abatement levels. Figure 4 shows best-response curves for the baseline setting with 4 inspections for 10 firms using the optimal allocation of 3 inspections for group 2 . The left panel shows the interaction between a group-1 firm and a group-2 firm, under the assumption that other six group-1 firms and the other two group-2 firms all abate at their equilibrium levels. The right panel shows the interaction between two group-2 firms, again assuming equilibrium behavior among the other eight firms.

Figure 4 shows that abatement effort is, for the most part, a strategic substitute; that is, if one firm increases its abatement effort, other firms best-respond by reducing theirs. The figure also reinforces the asymmetric behavior between targeted and untargeted firms, with targeted firms exerting much more abatement effort than untargeted ones. Not only does this occur in equilibrium, but also out of equilibrium. 
Figure 4. Best-response curves
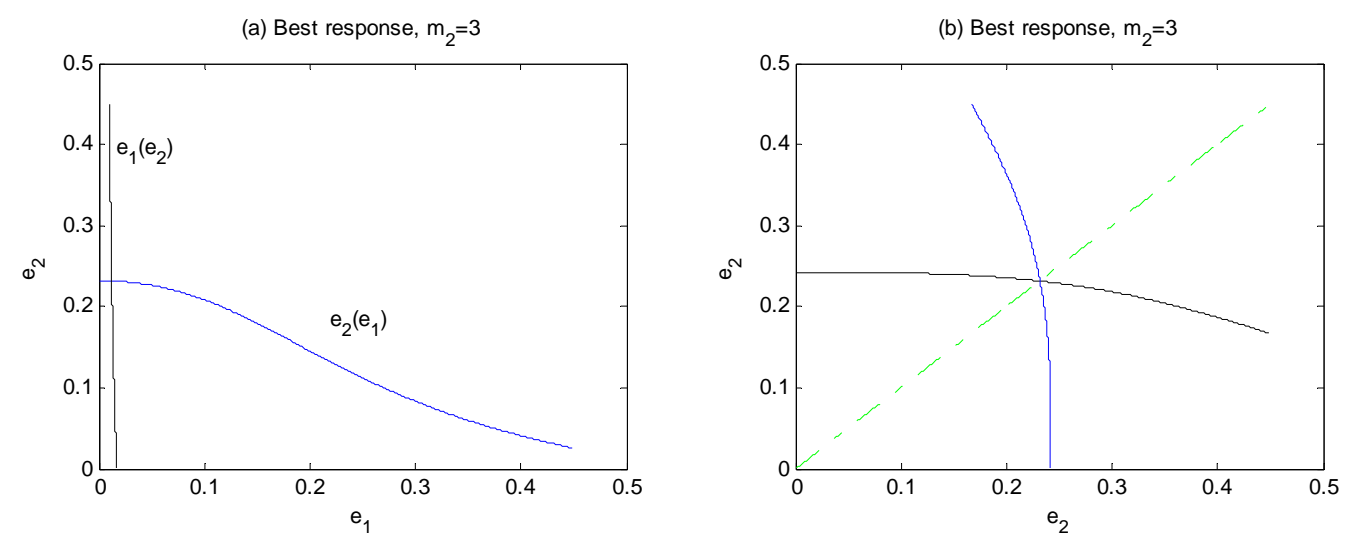

\section{Conclusion}

We develop a model of leveraged monitoring and enforcement when a regulator faces a fixed inspection capacity. The regulator places firms into two groups, a targeted group with a high inspection probability and an untargeted group with low inspection probability, and allocates the fixed number of inspections across those two groups. Inspected firms with the highest emissions are placed in the targeted group in the next period, and inspected firms with the lowest emissions are placed in the untargeted group in the next period. Uninspected firms remain in their original group. This mechanism invokes a rank-order tournament among the inspected firms, and the competition effect from the tournament enhances the leverage effect generated by the cost differential between the two groups.

Numerical simulations show that the regulator should allocate as many inspections to the targeted group as it allocates firms to the targeted group, so that firms in the targeted group are inspected with probability one. Also, about two-thirds of the total inspections should be allocated to the targeted group. However, even suboptimal allocations of firms and inspections to the untargeted group outperform static enforcement mechanisms in which all firms are placed into a single group and enforcement resources are not leveraged. 
The model presented in this paper is based on the assumption that a regulator faces a fixed inspection capacity in every period. How restrictive the inspection capacity is depends on the time horizon one considers. From a short-run perspective, the enforcement budget and the inspection personnel for a regulator are unlikely to change, and the effectiveness of the enforcement is confined by the limited number of inspections. In the long-run, the regulator may be able to adjust the budget or inspection staff according to actual firm behaviors. Still, the paper shows that the tournament imposed by the inspection capacity constraint enhances the leverage effect already identified in the literature. 


\section{Appendix A}

Table A1. Total abatement levels, 100 firms

\begin{tabular}{|c|c|c|c|c|c|c|c|c|c|}
\hline & \multicolumn{7}{|c|}{ Number of inspections in group 2 } \\
\hline $\begin{array}{c}\text { Total number } \\
\text { of inspections }\end{array}$ & 1 & 2 & 3 & 4 & 5 & 6 & 7 & 8 & 9 \\
\hline 4 & 0.2457 & 0.4747 & $\mathbf{0 . 6 0 8 2}$ & & & & & & \\
\hline 5 & 0.2564 & 0.4957 & $\mathbf{0 . 7 6 4 5}$ & 0.7087 & & & & & \\
\hline 6 & 0.2681 & 0.4883 & 0.8203 & $\mathbf{1 . 0 7 8 2}$ & 0.6866 & & & & \\
\hline 7 & 0.2701 & 0.4689 & 0.8394 & 1.1593 & $\mathbf{1 . 3 4 1 3}$ & 0.6110 & & & \\
\hline 8 & 0.3023 & 0.4492 & 0.8695 & 0.4451 & $\mathbf{1 . 5 8 4 2}$ & 1.3862 & 0.5363 & & \\
\hline 9 & 0.3266 & 0.4361 & 0.7264 & 1.1688 & 1.6193 & 1.9827 & $\mathbf{2 . 2 2 0 7}$ & 0.4842 & \\
\hline 10 & 0.3465 & 0.4324 & 0.6732 & 1.1034 & 1.6046 & 2.0696 & $\mathbf{2 . 2 2 0 7}$ & 1.0668 & 0.4572 \\
\hline
\end{tabular}

Note: bold numbers represent the total effort from the optimal allocations 


\section{Appendix B}

In this appendix, errors are uniformly distributed over [-0.5, 0.5] (except for the last graph, in which the range of the uniform distribution varies).

Table B1. Baseline example: 4 inspections for 10 firms

\begin{tabular}{|c|c|c|c|c|c|}
\hline$m_{2}$ & $e_{1}$ & $e_{2}$ & $n_{1} e_{1}$ & $n_{2} e_{2}$ & $n_{1} e_{1}+n_{2} e_{2}$ \\
\hline 3 & 0.0216 & 0.3173 & 0.1511 & 0.9518 & 1.1029 \\
\hline 2 & 0.0418 & 0.1674 & 0.3348 & 0.3348 & 0.6696 \\
\hline 1 & 0.0498 & 0.1308 & 0.4481 & 0.1308 & 0.5788 \\
\hline 0 & 0.0333 & -- & 0.3333 & -- & 0.3333 \\
\hline
\end{tabular}

Note: inconsistencies of calculation are due to rounding errors.

Figure B1. Robustness of the baseline simulation: 4 inspections for 10 firms
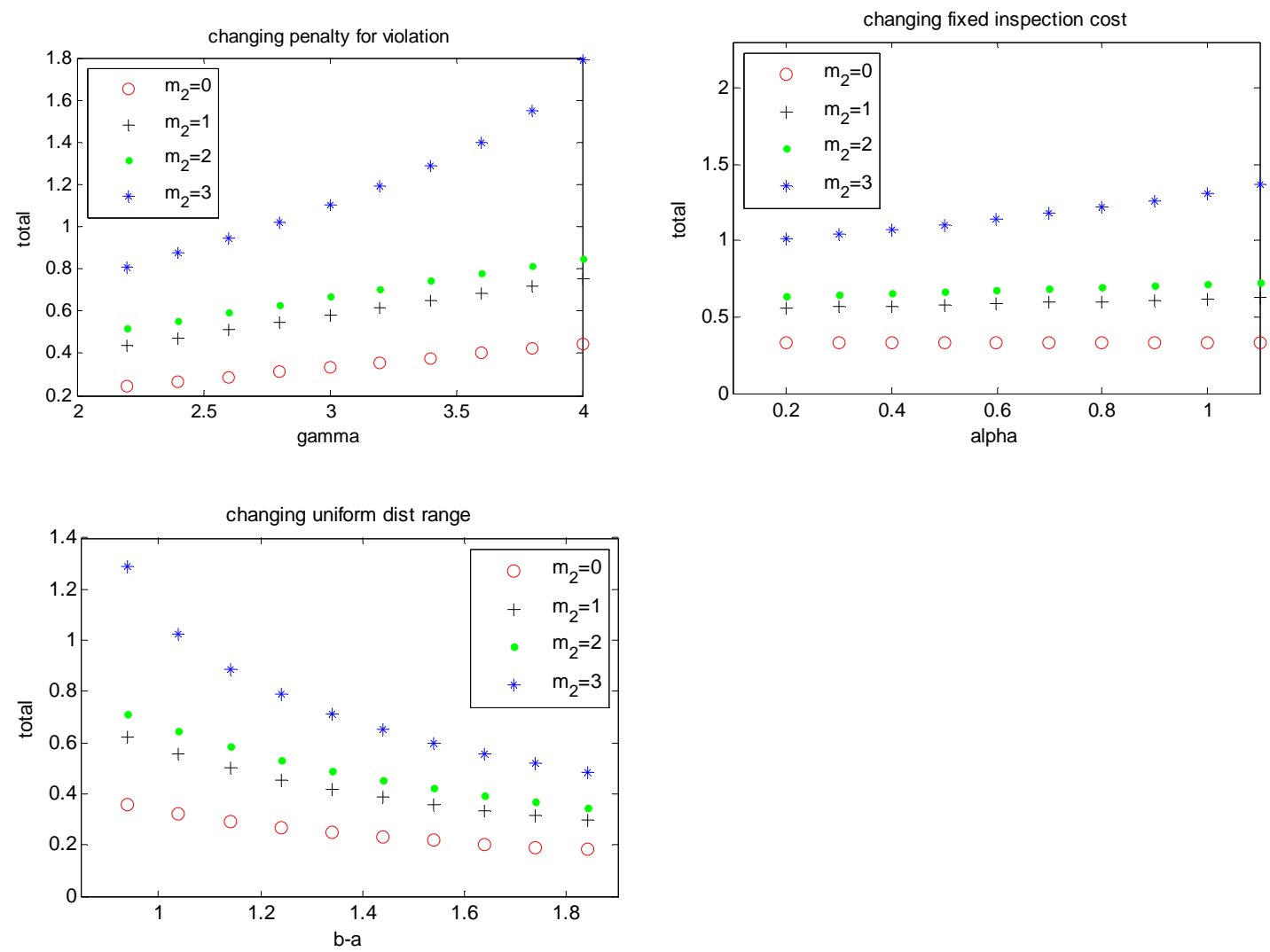


\section{References}

Franckx, L., D’Amato, A., 2004. Multitask rank order tournaments. Economics Bulletin 10, 110.

Friesen, L., 2003. Targeting enforcement to improve compliance with environmental regulations. Journal of Environmental Economics and Management 46, 72-85.

Govindasamy, R., Herriges, J.A., Shogren, J.F., 1994. Nonpoint tournaments, in Doswe, C., Tomasi, T. (Eds.), Nonpoint Source Pollution Regulation: Issues and Analysis. Kluwer Academic Publishers, Dordrecht, 87-106.

Harford, J.D., 1991. Measurement error and state-dependent pollution-control enforcement. Journal of Environmental Economics and Management 21, 67-81.

Harford, J.D., Harrington, W., 1991. A reconsideration of enforcement leverage when penalties are restricted. Journal of Public Economics 45, 391-395.

Harrington, W., 1988. Enforcement leverage when penalties are restricted. Journal of Public Economics 37, 29-53.

Jones, C.A., 1989. Standard setting with incomplete enforcement revisited. Journal of Policy Analysis and Management 8, 72-87.

Lazear, E.P., Rosen, S., 1981. Rank-order tournaments as optimum labor contracts. Journal of Political Economy 89, 841-864.

Nalebuff, B.J., Stiglitz, J.E., 1983. Prizes and incentives: towards a general theory of compensation and competition. The Bell Journal of Economics 14, 21-43.

Raymond, M., 1999. Enforcement leverage when penalties are restricted: a reconsideration under asymmetric information. Journal of Public Economics 73, 289-295.

Russell, C., 1990. Game models for structuring monitoring and enforcement systems. Natural Resource Modeling 4, 143-173. 
Stafford, S.L., 2008. Self-policing in a targeted enforcement regime. Southern Economics Journal 74, 934-951.

Viscusi, W. K., Zeckhauser, R., 1979. Optimal standards with incomplete enforcement. Public Policy 27, 437-456.

U.S. Bureau of the Census, 1999. Pollution Abatement Costs and Expenditures. US Government Printing Office, Washington, DC.

U.S. Environmental Protection Agency, 2004. Strategic Plan Region 4, EPA website http://www.epa.gov/cfo/regionplans/ region4/2004strategicplanreg4.pdf. 